\title{
Classification and characterization of human full-length cDNA clones that are difficult to sequence
}

\author{
Akihiko Kishimoto $^{1 *}$, Yukio Ono ${ }^{2 \dagger}$, Katsuji Murakawa ${ }^{2 \#}$, Tadashi \\ Ishibashi $^{2 \dagger}$, Ai Wakamatsu ${ }^{3 \ddagger}$, Keiichi Kanehori ${ }^{2 \&}$, Nobuo Nomura ${ }^{4}$, Takao \\ Isogai $^{3 \star}$, Masafumi Yohda ${ }^{5}$, and Sumio Sugano ${ }^{6 \S}$ \\ ${ }^{1}$ Hitachi, Central Research Laboratory, 1-280 Higashi-koigakubo, Kokubunji, Tokyo 185-8601, Japan \\ ${ }^{2}$ Hitachi Science Systems, 1-280 Higashi-koigakubo, Kokubunji, Tokyo 185-8601, Japan \\ ${ }^{3}$ Helix Research Institute, 1532-3 Yana, Kisarazu, Chiba 292-0812, Japan \\ ${ }^{4}$ BIRC, AIST, 2-42 Aomi, Koto-ku, Tokyo 135-0064, Japan \\ ${ }^{5}$ Tokyo University of Agriculture and Technology, 2-24-16, Naka-cho, Koganei, Tokyo 184-8588, Japan \\ ${ }^{6}$ The Institute of Medical Science, The University of Tokyo, 4-6-1 Shirogane-dai, Minato-ku, Tokyo \\ 108-8639, Japan \\ Present address: \\ ${ }^{\dagger}$ Hitachi High-Tech Manufacturing \& Service Corporation, 1040, Ichige, Hitachinaka-shi, Ibaraki-ken \\ 312-0033, Japan \\ \#Hitachi, Central Research Laboratory, 1-280 Higashi-koigakubo, Kokubunji, Tokyo 185-8601, Japan \\ ${ }^{*}$ Graduate School of Pharmaceutical Sciences, The University of Tokyo, 7-3-1 Hongo, Bunkyo-ku, Tokyo \\ 113-0033, Japan \\ ${ }^{\&}$ Quantum 14 KK, Tokyo University of Agriculture and Technology, 1204 Incubation Bldg. 2-24-16 \\ Naka-cho, Koganei-shi, Tokyo, 184-8588, Japan \\ ${ }^{\S}$ Laboratory of Functional Genomics, Department of Medical Genome Sciences, Graduate School of \\ Frontier Sciences, The University of Tokyo, 4-6-1 Shiroganedai, Minato-ku, Tokyo 108-8638, Japan \\ *E-mail: akihiko.kishimoto.hc@hitachi.com
}

(Received December 19, 2007; accepted February 6, 2008; published online March 2, 2008)

\begin{abstract}
In the Full-length Human cDNA Sequencing Project, 30,160 cDNA were sequenced. Among them, our group performed sequencing of 3,588 cDNAs, mainly using the primer walking method. The sequences achieved an average Phrap score of 76 , which means the average of expected sequence accuracy was $99.9999975 \%$, by sequencing of both strands with the criterion of a Phrap score over 30. In spite of the extremely high sequence reliability, we met with difficulty in sequencing 52 cDNAs, which are termed undecipherable cDNAs. cDNAs of long repeats were considered as a possible source of sequencing difficulty; their maximum repeat length sequenced by the primer walking method was $530 \mathrm{bp}$, without using the random method, and $81 \%$ of long repeat sequences remained in the ORFs. In single repeat regions, the insertion/deletion rates were much larger than in the usual regions. The fraction of SINE/Alu repeats in the cDNAs was $5.4 \%$, half of the fraction of the human genome. The fraction of SINE/Alu in undecipherable cDNAs was up to $10 \%$, the same level of the human genome.
\end{abstract}

Key Words: full-length cDNA, difficult to sequence, primer walking method, measurement accuracy, Phrap score

Area of Interest: Genome Wide Experimental Data Analyses 


\section{Introduction}

Full-length cDNAs, which are complete copies of mRNAs, are extremely important not only for determining gene structures in the genome, but also for practical applications. Using full-length cDNAs obtained by the oligo capping method [1] and long cDNAs obtained with the size fractionation method [2] as sources, the "Full-length Human cDNA Sequencing Project" (FLJ project) was conducted from April 1999 to March 2002. Finally, cloning and sequencing of 30,160 cDNA clones were performed [3].

Based on the accumulation of full length cDNA sequences, Full-length cDNA Annotation Invitational (H-invitational) was held in Tokyo and annotation for these sequences was performed. At that time, the FLJ project registered 21,243 cDNAs [3], which was almost half of the total entry of 42,469 cDNAs [4][5] registered in public databases through the DNA Data Bank of Japan (DDBJ). The annotated H-Invitational Database (H-Inv DB) is also publicly available [6], and the number of entry transcripts reached 175,539 on September 27th 2007 release 4.6. 120,558 human mRNA extracted from public databases in addition to 54,978 human full-length cDNAs, available on June 15, 2006. H-Inv DB includes SNPs (Single Nucleotide Polymorphisms) annotation, Representative Alternative Splicing Variant functional annotation, etc. Sequenced full-length human cDNA clones in FLJ project were distributed by NITE (National Institute of Technology and Evaluation; http://www.nite.go.jp/). These information and cDNA clones may contribute to the progress of genomic and proteomic research.

In large sequencing projects, quality control of the sequence data is extremely important, because accuracy of the sequence data is essential for the selection of novel cDNA clones, determination of protein coding regions, identification of variants such as SNPs or haplotypes, comparison of genome interspecies, and finding disease genes. In this paper, sequence accuracy of 3,588 cDNAs sequenced by the Hitachi Science Systems Team in the FLJ project is first discussed. Then, some cDNAs that were difficult to sequence are classified and analyzed.

\section{Materials and Methods}

\subsection{Full length cDNA clones}

All cDNA clones used in this paper were obtained from full-length cDNA libraries constructed by oligo capping method [1]. The cDNA clones were isolated at the Helix Research Institute and The Institute of Medical Science, The University of Tokyo. Single pass sequences of 5'-end of full-length cDNAs [7] were analyzed and then the complete and novel cDNAs were selected by comparison with public cDNA data [8]. The average insert size of the cDNAs was about $2.5 \mathrm{kbps}$.

E. coli cells containing cDNAs were cultivated in $3 \mathrm{~mL}$ of Luria-Bertani medium at $37^{\circ} \mathrm{C}$ for 15 hours. Extraction of plasmid DNAs was performed using a plasmid DNA isolation system (PI-1005: Kurabo industries, Osaka, Japan). Purity and the insert length of cDNA clones were analyzed by agarose gel electrophoresis.

\subsection{Sequencing}

cDNA sequences were determined by Dye-terminator method using the BigDyeTerminator Cycle Sequencing Kit (BDT) (Applied Biosystems, Foster City, CA), dGTP BigDyeTerminator 
Cycle Sequencing Kit (dGBDT) (Applied Biosystems), Thermo Sequenase II Terminator Cycle Sequencing Kit (TS) (Amersham-Pharmacia Biotech, Buckinghamshire, England), and DYEnamic ET Terminator Cycle Sequencing Kit (ET) (Amersham-Pharmacia Biotech). Among these, BDT was mainly used. Sequence reactions were performed according to the protocols supplied with the kits. If necessary, glycerol, DMSO (dimethyl sulfoxide), or SEQUENCHER Rx (Invitrogen, Carlsbad, CA) was added to the reaction mixtures.

Sequencing was mainly performed using a capillary array DNA sequencer, ABI Prism 3700 (Applied Biosystems / Hitachi). The capillary length was $50 \mathrm{~cm}$ and POP6 (Applied Biosystems) was used as filling polymer. The capillary temperature was $50^{\circ} \mathrm{C}$, and the temperature of the cuvette in the detection chamber was set to be optimal between 40 to $46^{\circ} \mathrm{C}$ for each sample. The reaction products obtained with dGBDT or TS were analyzed on a slab gel type electrophoresis 377 DNA-Sequencer (Applied Biosystems). The slab gel was adjusted $48 \mathrm{~cm}$ in length, 4\% polyacrylamide.

Full-length cDNA sequences were mainly obtained through the primer walking method. All sequences were confirmed by sequencing both strands. Other sequencing methods, such as transposon or shotgun methods were applied if necessary. Subclones containing required regions were used for sequencing. The transition reaction was performed according to the manufacturer's protocol of the kit (EZ::TN<KAN-2>Insertion Kit, EPICENTRE Biotechnologies, Madison, Wisconsin).

\section{Results}

\subsection{Sequencing accuracy}

Our group performed sequencing on 3,588 cDNAs out of the total 30,160 cDNAs sequenced in the FLJ project. The total number of sequenced nucleotides was $8,874,594 \mathrm{bp}$. The average and maximum length of the cDNAs was $2,473 \mathrm{bp}$ and $6,434 \mathrm{bp}$, respectively. The average GC content was $48 \%$, ranging between $28 \%$ and $69 \%$ in the 3,588 cDNAs whose average size was $2.5 \mathrm{kbp}$. A Phrap score distribution [9][10] of 2,400,905bp out of 8,874,594bp is shown in Figure 1.

In the FLJ project, the final sequences were established by complete overlap of both strands. Furthermore, our group set the Phrap score criterion at over 30 for each nucleotide. Consequently, the average Phrap score of 76 was achieved, which means the average of the expected sequence accuracy was as high as $99.9999975 \%$. For these, $99.9 \%$ of the nucleotide sequences exhibited Phrap scores over 70 .

On the other hand, the average of the expected sequencing accuracy of draft sequence of international human genome project was $99.75 \%$ and its Phrap score was 26 [11], and that of the finished sequences was over 99.999\%, with an error rate of 1 event per 100,000 bases [12] [13] (Figure 1). The finished sequences satisfied the world standards for the sequence fidelity known as the Bermuda Standards (http://www.gene.ud.ac.uk/ hugo/ bermuda2.htm). 


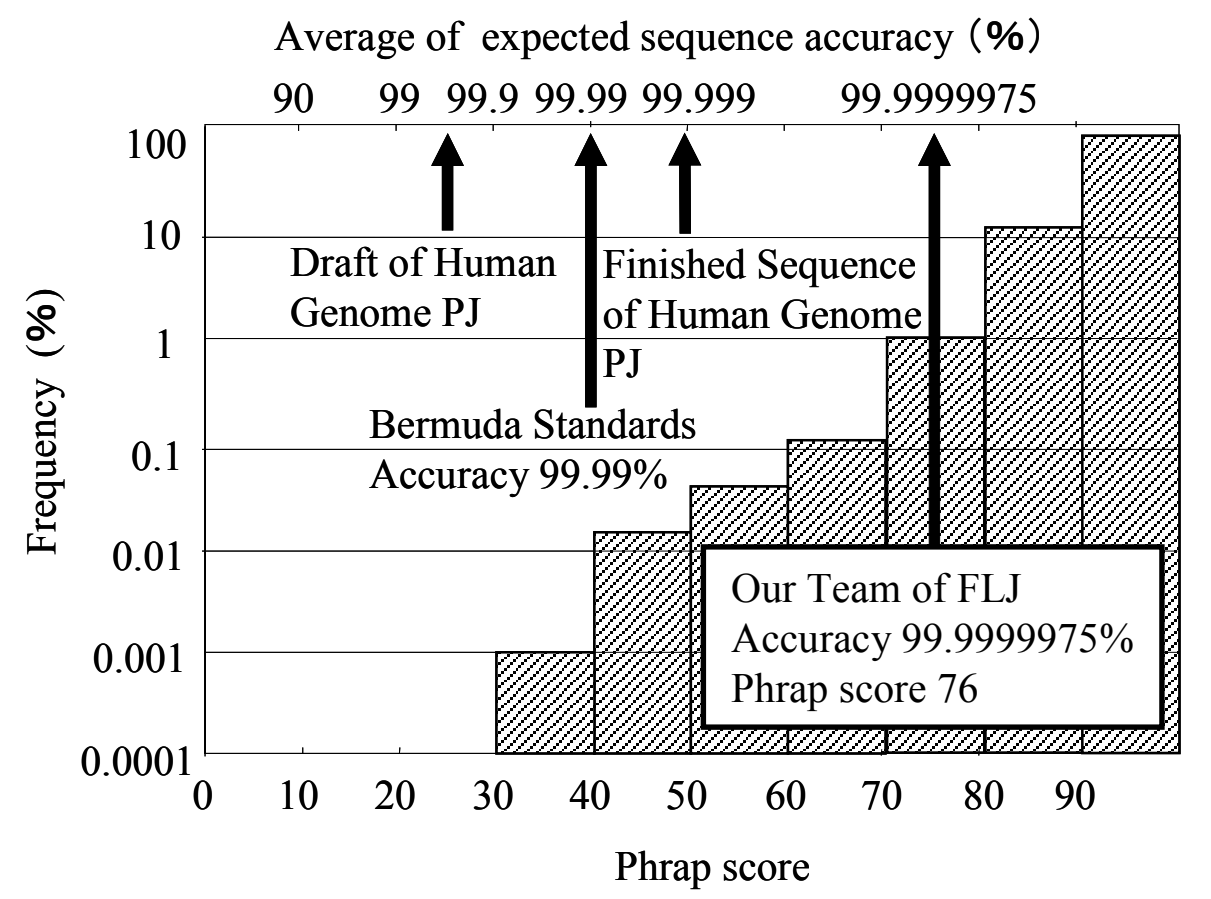

Figure 1. Frequency Distribution of Phrap score.

\subsection{Improvement of primer design method using PE number}

Despite the extremely high sequence accuracy expected in our project, sequences that are difficult to read still exist. As such we named these "undecipherable sequences". In our project, the primer walking method was predominantly used. At the beginning, primers were designed on melting temperature, secondary structure, GC content, free energy of 3' end, free energy of all the strands, and probability of hairpin structure formation. Primer designs were repeated until clear sequence results were obtained, but sometimes failed because of selection of an inadequate primer site.

In these cases, priming efficiency number (PE number) was introduced to design primers. PE number is a formulation included in the primer analysis software, OLIGO (Molecular Biology Insights, Inc., Cascade, CO), which quantifies the probability of priming at a given site on the template. The PE number is calculated by (1) the stability of primer-template duplexes and their distances from the 3' end; (2) bulge loops and mismatches and their distance from the 3' end; and (3) the overall primer length. To obtain high quality sequence data, the PE number of the primer should be high at the primer-starting region and low in whole sequences of the plasmid. PE numbers of the various primers used are plotted in Figure 2. Most of the primers that gave clear sequence results are plotted in the restricted region, where PE numbers for the sequencing start region and for the whole region are over 300 and below 200, respectively. On the contrary, primers with disordered sequence results are scattered all over the region. Using these criteria for designing primers, the failed primer rate decreased from $20 \%$ to $3 \%$. Thus, false priming was no longer a cause of the difficulty in sequencing of full length cDNAs.

\subsection{Classification of undecipherable cDNAs}

At the beginning of the project, 500 cDNA clones were sequenced. Among them, $36 \mathrm{cDNAs}$, $7.2 \%$ of the 500 cDNAs, were classified as undecipherable sequences. The main cause of the 
difficulty was false priming site, which was observed in 23 cDNAs or $4.6 \%$ of the 500 cDNAs. After the introduction of the PE number for designing primers, the number of undecipherable cDNAs was reduced to $52 \mathrm{cDNAs}$, to $1.4 \%$ of the $3,588 \mathrm{cDNAs}$. The causes and solutions for these undecipherable cDNAs are shown in Table 1. The causes were classified into long repeats, dinucleotide-rich, secondary structure or single nucleotide repeats. Tandem Repeats Finder [14] (http://tandem.bu.edu/trf/trf.html) was used to evaluate repeat sequences, and maximum score of repeats and its repeat length are shown in Table 1 . We searched each of the 52 undecipherable cDNAs against UniGene (http://www.ncbi.nlm.nih.gov/entrez/query.fcgi?db=unigene) [15] at the end of September, 2007. Thirty-five cDNAs had RefSeq [15] sequences in their cluster (indicated as "1"). Sixteen cDNAs did not include a RefSeq sequence (indicated as "0"), and one cDNA did not hit against the UniGene database. We searched using H-Inv DB and BLAST, and this cDNA was similar to FLJ36144 (indicated as "-1").

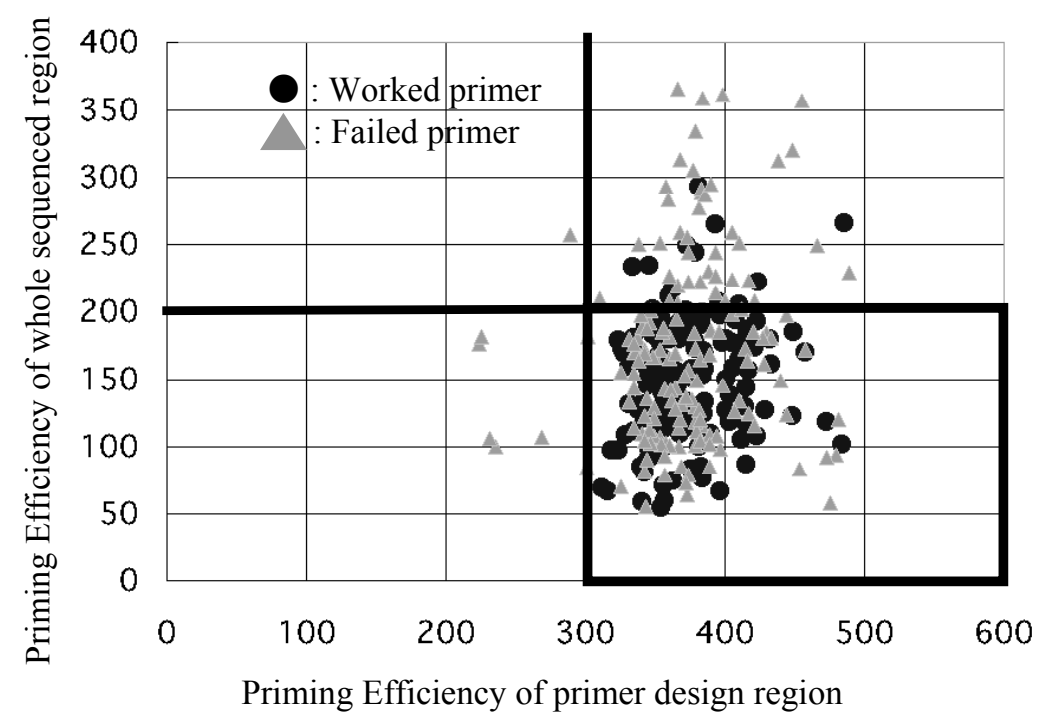

Figure 2. Primer Efficiency number of various primers.

\subsection{Undecipherable cDNAs with long repeats}

Among undecipherable cDNAs with long repeats, the cDNA, CTONG3002588, exhibited a low Tandem Repeats Finder score under 100. Yet, almost identical sequences of about 500 bp with a space of 1,000 bp were detected by Dotlet (http://myhits.isb-sib.ch/cgi-bin/dotlet) [16]. Next, we examined the sequence and found two $536 \mathrm{bp}$ repeat elements within $2116 \mathrm{bp}$. Among 20 undecipherable cDNAs with long repeats, 19 cDNAs were sequenced by random method such as shotgun or transposon techniques. Only one exception, BRTHA3013882, did not require sequencing by a random method. This cDNA has a high score of 706 with a pair of $530 \mathrm{bp}$ of GT repeat sequences. Lengths of the repeat sequences of the other undecipherable cDNAs were longer than $530 \mathrm{bp}$. Of these, $16 \mathrm{cDNAs}$ with long repeats contained ORFs and $13 \mathrm{cDNAs}$, namely $81 \%$, contained repeat sequence within ORFs. It seems that many repeat sequences remained in the ORFs, especially in undecipherable cDNA clones.

Next, we investigated a cDNA, TESTI2004080, as an example of an undecipherable cDNA 
Chem-Bio Informatics Journal, Vol. 8, No. 1, pp.1-13(2008)

Table 1. Causes and solutions of undecipherable cDNA.

\begin{tabular}{|c|c|c|c|c|}
\hline CloneName & Cause & Solution & repeat length & RefSeq \\
\hline BRTHA3013882 & Long repeat & ET, additive agent & 530 & 0 \\
\hline THYMU2008994 & Long repeat & Shotgun & 590 & 1 \\
\hline TSTOM 2000235 & Long repeat & Transposon & 603 & 1 \\
\hline TEST I2008762 & Long repeat & Shotgun & 630 & 1 \\
\hline THYMU2012919 & Long repeat & Shotgun & 630 & 1 \\
\hline PROST2005067 & Long repeat & Shotgun & 652 & 1 \\
\hline BRHIP3024703 & Long repeat & Transpozon, BDT, TS & 696 & 1 \\
\hline NT2RM4000634 & Long repeat & Shotgun & 932 & 0 \\
\hline TST02993 & Long repeat & Shotgun & 1068 & 0 \\
\hline BRHIP3017855 & Long repeat & Transpozon, BDT & 1086 & 1 \\
\hline TESTI2003940 & Long repeat & Shotgun & 1174 & -1 \\
\hline TESTI2002293 & Long repeat & Shotgun & 1175 & 1 \\
\hline PROST2008770 & Long repeat & Shotgun & 1252 & 1 \\
\hline BRHIP3025844 & Long repeat & Transpozon, BDT, dGBDT & 1343 & 1 \\
\hline NT2RI2009239 & Long repeat & Shotgun & 1360 & 0 \\
\hline TRACH3019290 & Long repeat & Transpozon, BDT & 1420 & 1 \\
\hline SPLEN2022162 & Long repeat & Shotgun & 1436 & 1 \\
\hline TESTI2004080 & Long repeat & Shotgun & 1573 & 1 \\
\hline TKIDN2011051 & Long repeat & Transpozon, BDT & 1690 & 0 \\
\hline CTONG3002588 & Long repeat & Transpozon, BDT & 2116 & 1 \\
\hline TESOP2009247 & GA-rich & $\mathrm{BDT}, \mathrm{dGBDT}$ & 30 & 1 \\
\hline NT2RP3000628 & GA-rich & $\mathrm{dGBDT}, 60^{\circ} \mathrm{C}$ reaction & 38 & 0 \\
\hline NT2RP3004670 & GC-rich & Shotgun & 68 & 1 \\
\hline PROST2007950 & GC-rich & Shotgun & 70 & 1 \\
\hline UTERU3021231 & GC-rich & Transpozon, BDT & 84 & 1 \\
\hline TLUNG2000654 & GC-rich & $\mathrm{BDT}, \mathrm{dGBDT}$ & 97 & 1 \\
\hline NT2RP3000512 & GC-rich & $\mathrm{dGBDT}, 60^{\circ} \mathrm{C}$ reaction & 124 & 1 \\
\hline TESTI2026024 & GC-rich & Transpozon, BDT, dGBDT & 133 & 1 \\
\hline BRHIP3035222 & GT/GA-rich & dGBDT, TS, additive agent & 348 & 0 \\
\hline TESTI2037085 & GT/GA-rich & dGBDT, TS, additive agent & 382 & 0 \\
\hline SLV06258 & GC-rich & $\mathrm{BDT}, \mathrm{dGBDT}$ & 437 & 1 \\
\hline BRHIP3032413 & GC-rich & Transpozon, BDT & 583 & 1 \\
\hline BRHIP3020155 & GC-rich & Transpozon, BDT & 602 & 1 \\
\hline SMINT2017964 & CT-rich & Transpozon, BDT, dGBDT & 656 & 1 \\
\hline BRHIP3038037 & GC-rich & Transpozon, BDT & 900 & 1 \\
\hline SPL03856 & 2nd Structure & Transpozon, BDT, dGBDT & 0 & 1 \\
\hline TESTI2023386 & 2nd Structure & BDT, dGBDT & 29 & 0 \\
\hline TESTI2005153 & 2nd Structure & Transpozon, BDT, dGBDT & 30 & 1 \\
\hline SPL00128 & 2nd Structure & BDT, dGBDT & 31 & 1 \\
\hline SLV05412 & 2nd Structure & Transpozon, BDT, dGBDT & 40 & 0 \\
\hline FCBBF3026692 & 2nd Structure & Shotgun & 48 & 1 \\
\hline NT2RP3001383 & 2nd Structure & TS, dGBDT & 58 & 1 \\
\hline BRTHA3013860 & 2nd Structure & Sonication, Shotgun & 64 & 1 \\
\hline Y79AA1000346 & 2nd Structure & Determine from R-strand & 71 & 1 \\
\hline BRACE3046855 & 2nd Structure & $\mathrm{BDT}, \mathrm{dGBDT}$ & 195 & 0 \\
\hline SPL05416 & 2nd Structure & Transpozon, BDT, dGBDT & 279 & 0 \\
\hline BRHIP3036371 & 2nd Structure & Transpozon, BDT, dGBDT & 562 & 1 \\
\hline NT2RM4002504 & Single repeat & Shotgun & 26 & 0 \\
\hline NT2RP3000418 & Single repeat & Shotgun & 26 & 0 \\
\hline THYRO1001480 & Single repeat & TS, dGBDT & 35 & 0 \\
\hline TRACH2011057 & Single repeat & Transpozon, TS & 60 & 1 \\
\hline SYN00365 & Single repeat & Shotgun & 89 & 0 \\
\hline
\end{tabular}


containing long repeats within the ORF. This cDNA was annotated as polyubiquitin. Figure 3 shows the overview of repeats of this cDNA examined by Dotlet. The Tandem Repeats Finder detected two repeat sequences between $145 \mathrm{bp}$ to 1,773 bp. Longer sequences repeated seven times, with a repeat element length of $228 \mathrm{bp}$, and shorter ones repeated twice, with a repeat element length of 76bp. There are $62 \mathrm{bp}$ differences, $4 \%$ of the overall repeat sequences, in the consensus sequence among the repeat sequences. Yet, these nucleotide variations did not cause any amino acid sequence change. Then, we compared the similarity of repeat sequences of this cDNA with that of GenBank using BLAST. For this, 11 cDNAs were selected from the searches using a bit score [17] criterion at over 1,000. Amino acid sequence in repetition remained unchanged not only within this TESTI2004080, but also in the other similar cDNAs in the public database. This is consistent with the notation that the ubiquitin sequence is conserved. Undecipherable cDNAs caused long repeats with repeat lengths over $500 \mathrm{bp}$, clear repeats were observed, and repeats were usually within ORFs.

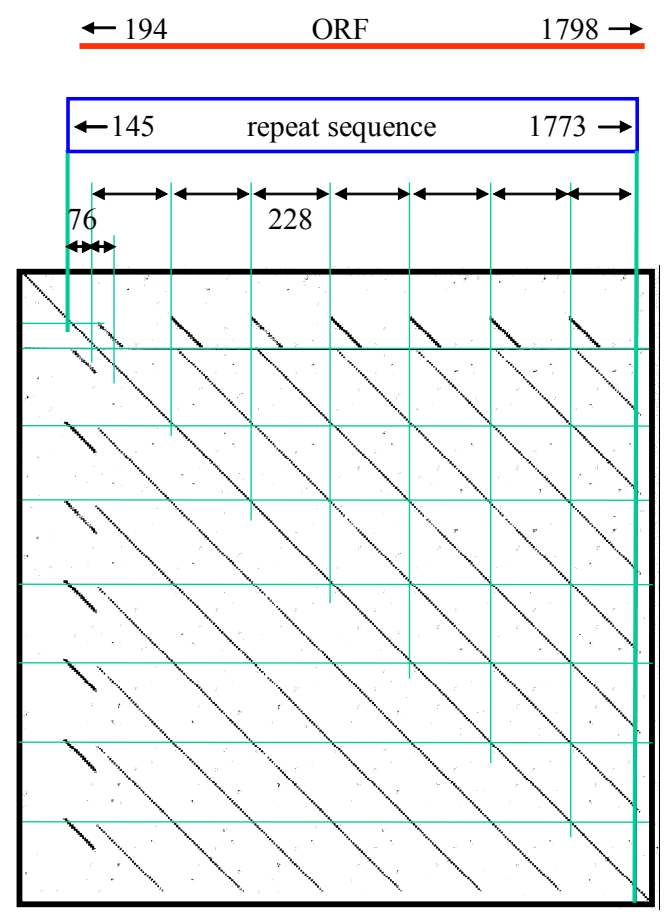

Figure 3. An overview of repeat sequences of TESTI2004080 through Dotlet.

\subsection{An example of undecipherable cDNAs with hairpin structure}

Figure 4 shows the sequence chromatograms of a cDNA BRTHA3013860. As detected by the sequence chemistry of BDT, peak intensity decreased suddenly after GGGAGGGGTC (Figure 4(a)). Thus, we changed the sequence chemistry to dGBDT. Although the chromatogram was improved, there the difficulty in base calling still remained (Figure 4 (b)). In addition, signal intensity decreased in a single adenosine repeat region, which was 180 bases after this GC-rich region (data not shown). This was likely due to the formation of secondary structure between GGGAGGGGTC and a C-rich region found in the newly readable region. Thus, the cDNA was sequenced by the shotgun method using TS chemistry. With this method, the disorder of the sequence disappeared (Figure 4 (c)), and signal intensity in the single adenosine repeat region was also improved. 
Figure 5 shows the result of conformation prediction for this sequence from BRTHA3013860, using a secondary structure analysis program, RNAfold (http://rna.tbi.univie.ac.at/) [18]. An arm of hairpin structure consisting of GGGAGGGGUC was coincident with the end of readable sequence in Figure 4 (a), and a tip of the hairpin, composed of GGCGU, corresponded to the place where the peaks disappeared. The arm of this hairpin consisted of almost $10 \mathrm{GC}$ pairs, which made the arm structure tight.

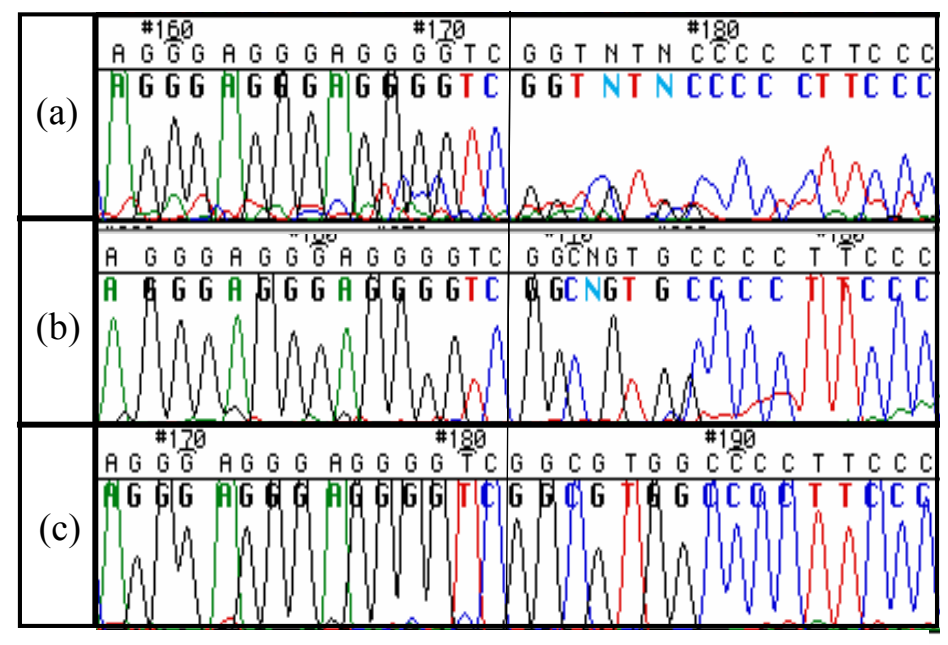

Figure 4. Sequence chromatograms of a cDNA, BRTHA3013860.

(a) BDT : BigDyeTerminator Cycle Sequencing Kit

(b) dGBDT : dGTP BigDyeTerminator Cycle Sequencing Kit

(c) TS : Thermo Sequence II Terminator Cycle Sequencing Kit

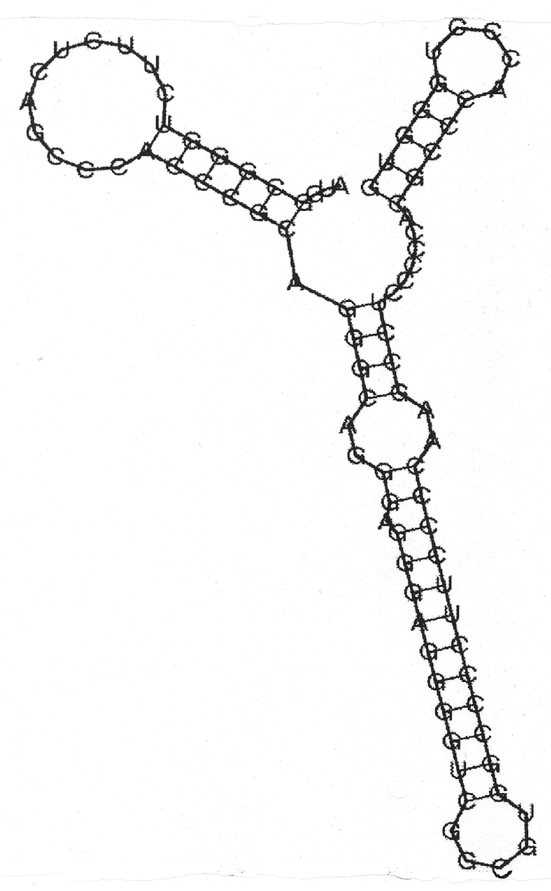

Figure 5. Conformation prediction of BRTHA3013860. 


\subsection{Simple sequence repeats in undecipherable cDNAs}

In addition to the poly-A tail, single nucleotide repeats often exist in cDNAs. These single nucleotide repeats often cause difficulty in sequencing. The chromatogram of a cDNA containing an A-rich region, THYRO1001480, is shown in Figure 6. Figure 6(a) shows that the peaks fall into disorder just after the T-rich region, which is the reverse strand data of the A-rich region. TS or dGBDT chemical reaction did not improve the chromatogram. Finally, we were able to obtain a clear sequence result using a primer designed in the T-rich region (Figure 6(b)).

Dotlet analysis of THYRO1001480 is shown in Figure 7, and is consistent with the result obtained by RepeatMasker. The A-rich region is located in the end of SINE/Alu sequence. In the cDNA, there are five SINE/Alu sequences of about 300 bp of length, which locate at 62-373, 776-1084, 1255-1558, 1694-2003, 2021-2331 bps respectively. A-rich regions are observed in 344-381, 1057-1084, 1974-2008, 2302-2340 bps, which were detected as single nucleotide repeats by Tandem Repeats Finder. One of the SINE/Alu sequences is inverted, and does not have an A-rich region [19]. The undecipherable region shown in Figure 6 corresponds to the A-rich region in 1974-2008 bps. Interestingly, THYRO1001480 has a very genome-like character, because $99 \%$ of the sequences were composed of SINE/Alu and LINE/L1 elements.

There are 4 insertion/deletions in this cDNA. Three of them are in A-rich regions located at the end of SINE/Alu elements. The undecipherable sequence in 1974-2008 bps is also included.

We performed a BLAST search with each of the other four undecipherable cDNAs, whose sequencing difficulties were caused by single nucleotide repeats (Table 2). Among them, a cDNA, SYN00365, was annotated as an immunoglobulin lambda heavy chain, which has various DNA gene segments that are spliced from the chromosome, and thus, we excluded it from the objects. Consequently, out of eight sequences homologous to cDNAs that were undecipherable due to single repeats, six sequences were not coincident with the undecipherable cDNAs. So, the insertion/deletion rates in single repeat regions were much larger than in the usual regions.

(a)

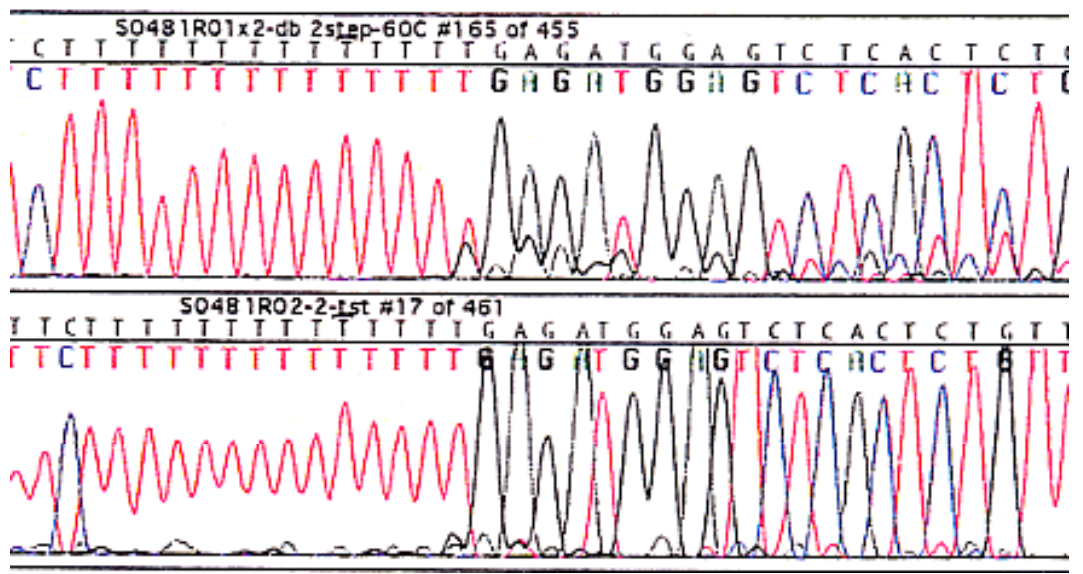

Figure 6. Chromatogram of a cDNA containing A-rich region, THYRO1001480.

(a) Primer was designed outside of T-rich region of the reverse strand.

(b) Primer was designed inside of T-rich region of the reverse strand. 


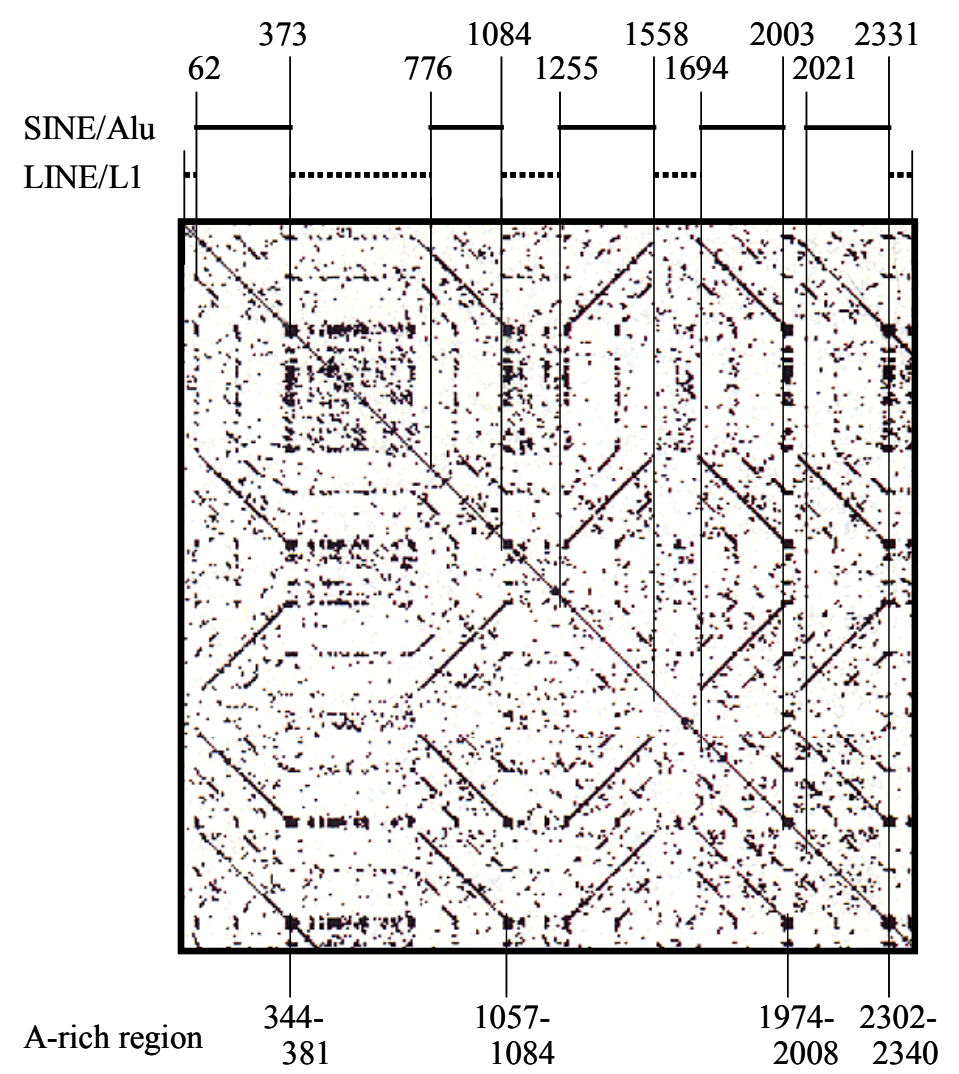

Figure 7. An overview of SINE/Alu and LINE/L1 of THYRO1001480 by Dotlet.

Table 2. Number of repeat bases of undecipherable cDNAs, whose sequencing difficulties were caused by single nucleotide repeats.

\begin{tabular}{|c|c|c|c|}
\hline FLJ cDNA name & FLJ & Hit & notes \\
\hline \multirow{3}{*}{ THYRO1001480 } & 30 & 30 & $342-371 \mathrm{bp}$ \\
\cline { 2 - 4 } & 28 & 29 & $1057-1084 \mathrm{bp}$ \\
\cline { 2 - 4 } & 35 & 34 & $1974-2008 \mathrm{bp}$ \\
\cline { 2 - 4 } & 39 & 38 & $2302-2340 \mathrm{bp}$ \\
\hline \multirow{2}{*}{ TRACH2011057 } & 61 & 61 & altenative assembly \\
\cline { 2 - 4 } & 61 & 58 & reference assembly \\
\hline NT2RM4002504 & 18 & 19 & \\
\hline NT2TP3000418 & 26 & 25 & \\
\hline SYN00365 & 89 & - & Immunoglobulin \\
\hline
\end{tabular}

\subsection{Interspersed repeats in undecipherable cDNAs}

We analyzed interspersed repeats in the 3,588 cDNAs using RepeatMasker. The total number of base pairs in the 3,588 cDNA is $8,875 \mathrm{kbp}$, which is equivalent to about $0.3 \%$ of the human genome. The fraction of interspersed repeats in the $3,588 \mathrm{cDNAs}$ is $17 \%$ and in undecipherable cDNAs, it is 
$26.4 \%$. Their classification is shown in Table 3. Human genome data [11] were also analyzed for comparison. We observed that the fraction of SINE/Alu in the 3,588 cDNAs (10.3\%) is significantly different from that in undecipherable cDNAs $(5.4 \%)$ according to the chi-square test $(\mathrm{P}=7.53 \mathrm{E}-08)$. The fraction of simple repeats is also a highly significant difference in both cDNAs $(\mathrm{P}=6.57 \mathrm{E}-46)$. Average length of simple repeats in undecipherable cDNAs is $184 \mathrm{bp}$, and this is longer than that in the 3,588 cDNAs of $59 \mathrm{bp}$.

(A)n of simple repeats classified in interspersed repeats was same criterion of single repeats in undecipherable cDNAs. (CA)n, (TG)n, (CGG)n or (CCCGG)n of simple repeats correspond to dinucleotide-rich in undecipherable cDNAs. Simple repeats of long repeat length correspond to long repeats in undecipherable cDNAs. So it is reasonable that the fraction of simple repeat in undecipherable cDNAs is higher than in the 3,588 cDNAs.

According to a previous report [20], SINE/Alus were mostly seen in the intronic regions, occupying $12.8 \%$ of these areas. In addition, they were rarely found in exons, with total SINE/Alu coverage of exons being $1.6 \%$. As the fraction of ORF region in the 3,588 cDNA was $41.3 \%$ in our results, it is reasonable that the fraction of SINE/Alu in the 3,588 cDNA was half of the fraction of the genome.

SINE/Alu is a so called "retro transposon", and transfers as a "copy and paste" type, making it easy to induce repeat sequences. Usually, SINE/Alu had an A-rich region at the end of the sequence, which may become the cause of undecipherable cDNAs.

Table 3. Fraction of interspersed repeats in the human genome, the 3,588 cDNAs and undecipherable cDNAs.

\begin{tabular}{|c|c|c|c|c|c|c|}
\hline \multicolumn{2}{|c|}{ Sequence } & LINE/L1 & SINE/Alu & Simple repeat & Others & Total \\
\hline Human genome & $\mathrm{bp} \%$ & $16.9 \%$ & $10.6 \%$ & $3.0 \%$ & $17.3 \%$ & $47.8 \%$ \\
\hline (1)cDNAs & $\mathrm{bp} \%$ & $3.5 \%$ & $5.4 \%$ & $0.5 \%$ & $7.6 \%$ & $17 \%$ \\
\hline \multirow{2}{*}{ (2)undecipherable cDNAs } & $\mathrm{bp} \%$ & $4.0 \%$ & $10.3 \%$ & $4.1 \%$ & $7.9 \%$ & $26.4 \%$ \\
\cline { 2 - 7 } & number & 20 & 67 & 32 & 78 & 197 \\
\hline \multicolumn{2}{|c|}{ chi-test P(1)=(2) } & 0.550 & $7.53 \mathrm{E}-08$ & $6.57 \mathrm{E}-46$ & 0.732 & $5.94 \mathrm{E}-50$ \\
\hline
\end{tabular}

\section{Discussion}

\subsection{Maximum length of the primer walking method}

Sequencing here was primarily performed with the primer walking method. In the primer walking method, the next primer is usually designed from the last part of the previous read. If repeat length is longer than the read length, the previous read is full of repeat sequence, such that the primer can not be designed because there is no specific part in the previous read where the primer can hybridize. In such a case, we used a random method, such as shotgun or transposon sequencing. Causes and solutions for undecipherable cDNAs are shown in Table 1. According to this table, undecipherable cDNAs of long repeats (except BRTHA3013882), were sequenced using the random method. The repeat length of BRTHA3013882 was $530 \mathrm{bp}$. This length was the maximum sequence length in undecipherable cDNAs for only using the primer walking method and without using the random method. This length is almost equal to read length of $500 \mathrm{bp}$.

In Table 1 , the main cause of undecipherable cDNAs is indicated, but actually a complex cause 
could also be possible. In the case of dinucleotide-rich sequences, the rate of repeat length over 530 bp was $50 \%$, greater than the case of secondary structure, which was $14 \%$. If there is nucleotide species deviation in the repeat sequences, it is possible that it is the cause of the dinucleotide-rich region. Thus, causes of long repeats and dinucleotide-rich repeats may be easily coupled. On the other hand, sequence length of secondary structure may be relatively short, such that causes of long repeat and secondary structure are almost independent. Actually, in the case of repeat length it is shorter than read length, namely $530 \mathrm{bp}$, there are $14 \mathrm{cDNAs}$ which could not be sequenced primer walking method. These cDNAs may have a strong secondary structure (hard stop), such that if the read length is getting longer, there still remain some cDNAs that cannot be sequenced by the primer walking method.

\subsection{Reaction chemistry and random method for secondary structure}

Figure 4 shows an example of the progression of methods: first, the usual primer walking method of BDT reaction chemistry; second, the dGBDT for GC-rich suitable chemistry; and finally, the shotgun of random method.

When we found GC-rich or secondary structure regions, we managed to sequence by changing the selection of priming site or sequence reaction chemistry. In the GC-rich region, there was secondary structure of hydrogen bonds. For this, we tried adding dimethyl sulfoxide (DMSO) to resolve this issue. When we sequenced long A-rich or TC-rich regions using BDT reaction chemistry, slippage prevented a clear chromatogram. For these sequences, we adopted TS (Thermo Sequenase II Terminator Cycle Sequencing Kit), which uses an enzyme, thermo sequenase, for a slab gel type electrophoresis DNA Sequencer. Moreover, ET (DYEnamic ET Terminator Cycle Sequencing Kit) for a 96 capillary type electrophoresis 3700 DNA analyzer was used to produce a satisfactory chromatogram.

Namely, in the case when a satisfactory sequencing chromatogram could not be obtained by the primer walking method due to long repeats, secondary structure or GC-rich regions or the like ones, we applied appropriate reaction chemistry for the primer walking method to sequence as long as possible. Then, we used a random method, such as shotgun or transposon sequencing, to cover these sequences where sequence could not be determined by the primer walking method.

As shown in Table 1, out of the five undecipherable cDNAs with single repeats, four cDNAs were sequenced by the random method. The random method was effective in determining single repeat regions. By random methods, sub clones were cleaved into small fragments to inhibit formation of secondary structure. In the case of THYRO1001480, the primer was designed within the T-rich region, so that a clear chromatogram was obtained. This is believed to have occurred because the primer hybridized in a single repeat region and blocked secondary structure formation.

We thank Keiichi Nagai, Tetsuo Nishikawa, Toshio Ota, Tomoyasu Sugiyama, Ryotaro Irie, Tetsuji Otsuki, Jun-ichi Yamamoto and Mitsuo Sekine for their supports of cDNA selection, useful discussion and much more. We thank Osamu Ohara, Takahiro Nagase, Yutaka Suzuki, Yoshifumi Kawamura and Kouichi Kimura for their valuable discussions. We thank Hideki Kambara, for his helpful encouragement. We thank Tsugio Matsuo, Yasukatsu Miyoshi, Hisanori Ezoe and Katsufumi Takahashi in Research Association for Biotechnology for supporting this project. This work was supported by a Grant from NEDO of the Ministry of Economy, Trade and Industry of Japan. 


\section{References}

[1] K. Maruyama, S. Sugano, Oligo-capping: a simple method to replace the cap structure of eukaryotic mRNAs with oligoribonucleotides. Gene, 138, 171-174 (1994).

[2] N. Nomura, N. Miyajima, T. Sazuka, Prediction of the Coding Sequences of Unidentified Human Genes. I. The Coding Sequences of 40 Neq Genes (KIAA0001-KAA0040) Deduced by Analysis of Randomly Sampled cDNA Clones from Human Immature Myeloid Cell Line KG-1, DNA Res., 1, 27-35 (1994).

[3] T. Ota, Y. Suzuki, T. Nishikaswa et al., Complete sequencing and characterization of 21,243 full-length human cDNAs. Nature Genet., 36, 40-45 (2003).

[4] Mammalian Gene Collection (MGC) Program Team. Generation and initial analysis of more than 15,000 full-length human and mouse cDNA sequences, Genetics, 99, 26, 16899-16903 (2002).

[5] S. Wiemann, B. et al., Toward a catalog of humangenes and protein sequencing and analysis of 500 complete protein human cDNAs, Genome Res., 11, 422-435 (2001).

[6] Tadashi Imanishi et al., Integrative Annotation of 21,037 Human Genes Validated by Full-Length cDNA Clones, PLOS Biology, 2, 0001-0020 (2004).

[7] Kouichi Kimura, Ai Wakamatsu, Katsuji Murakawa, Takao Isogai, Sumio Sugano et al. Diversification of transcriptional modulation: Large-scale identification and characterization of putative alternative promoters of human genes, Genome Res., 16, 55-65 (2006).

[8] T. Nishikawa, T. Ota, Y. Kawai, Comparison of Sequences of cDNA Clones Obtained from Oligo-capping cDNA Libraries with Those from UniGene, DNA Res., 8, 255-262 (2001).

[9] B. Ewing, L. Hillier, M.C. Wendl and P. Green, Base-calling of automated sequencer traces using phred. I. Accuracy assessment, Genome Res., 8, 175-185(1998).

[10] B. Ewing, and P. Green, Base-calling of automated sequencer traces using phred. II. Error probabilities, Genome Res., 8, 186-194 (1998).

[11] International Human Genome Sequencing Consortium, the Human Genome. Nature, 409, 860-921 (2001).

[12] International Human Genome Sequencing Consortium, Finishing the euchromatic sequence of the human genome. Nature, 431, 931-945 (2004).

[13] Jeremy Schmutz, et al., Quality assessment of the human genome sequence, Nature, 429, 365-368 (2004).

[14] B. Gary, Tandem repeats finder; a program to analyze DNA sequences. Nucleic Acids Res. 27, 573-580 (1999).

[15] D. L. Wheeler, et al., Database Resources of the National Center for Biotechnology. Nucleic Acids Res. 31, 28-33 (2003).

[16] T. Junier and M Pagni, Dotlet: diagonal plots in web browser. Bioinformatics, 16, 178-179 (2004).

[17] S. F. Altschul, et al., Gapped BLAST and PSI-BLAST: a new generation of protein database search programs. Nucleic Acids Res. 25, 3389-3402 (1997).

[18] I. L. Hofacker, Vienna RNA secondary structure server. Nucleic Acids Res. 31, 3429-3431 (2003)

[19] K. S. Lobachev, et al., Inverted Alu repeats unstable in yeast are excluded from the human genome. EMBO J., 19, 3822-3830 (2000).

[20] D. Grover, et al., Alu repeat analysis in the complete human genome: trends and variations with respect to genomic composition. Bioinformatics, 20, 813-817 (2004). 